\title{
The NOvA Experiment, The First 12 Months of Commissioning, Operations and Physics Data
}

\author{
A.Norman for the NOvA collaboration
}

Fermi National Accelerator Lab, Bativia, IL 60510

\begin{abstract}
.
The NOvA experiment is a long baseline neutrino experiment designed to make precision measurements of the oscillation probabilities for $v_{\mu} \rightarrow v_{e}$ and $v_{\mu} \rightarrow v_{\mu}$ for both neutrinos and anti-neutrinos. These measurements will provide new information on the neutrino mass hierarchy, improve our knowledge of whether $\theta_{23}$ is non-maximal and possibility provide information on the CP violating phase $\delta_{C P}$ of the PMNS neutrino mixing matrix. We present the observations of the first neutrino event in the NOvA far and near detectors along with data obtained during the first year of detector commissioning and operations. We use the data to demonstrate the detector's ability to identify electron and muon neutrino events and to reject cosmic ray induced backgrounds at a level of $4 \times 10^{7}: 1$. New estimates for the signal and background sensitivities of the NOvA experiment during the first year of full detector running are presented.
\end{abstract}

Keywords: NOvA, neutrino, mixing, mass hierarchy, $\theta_{13}, \theta_{13}$

PACS: $14.60 . \mathrm{Pq}$

\section{INTRODUCTION}

The NOvA experiment has been designed and constructed to answer the next generation of questions regarding the underlying structure of the neutrino sector. In particular the NOvA experiment has been designed to use the Neutrinos at the Main Injector (NuMI) accelerator complex at Fermi National Accelerator Lab, in an off-axis configuration to measure and compare the oscillations of neutrinos and anti-neutrinos. The experiment uses a 14 kton far detector located at the Ash River Laboratory site in northern Minnesota and a smaller functionally identical 300 ton near detector located at Fermilab to provide an $810 \mathrm{~km}$ baseline over which the neutrinos travel. The siting of the experiment's detectors have been specifically chosen to place them in an "off-axis" configuration where they are $14 \mathrm{mrad}$ off of the primary axis for the NuMI beam. This configuration produces a narrow energy spectrum for the resulting neutrinos that peaks near $2 \mathrm{GeV}$ as shown in Fig.1. This narrow energy spectrum in combination with the $810 \mathrm{~km}$ baseline places the L/E of the experiment at the first oscillation maximum of the primarily $v_{\mu}$ beam. This tuning of the experiment to the oscillation parameters simultaneously maximizes the $v_{\mu} \rightarrow v_{e}$ appearance signal and minimizes $v_{\mu} \rightarrow v_{\mu}$ survival probability.

The core NOvA oscillation measurements will take the form of the neutrino and anti-neutrino oscillation probabilities $\mathrm{P}\left(v_{\mu} \rightarrow v_{e}\right)$ and $\mathrm{P}\left(\bar{v}_{\mu} \rightarrow \bar{v}_{e}\right)$ as well as the survival probabilities $\mathrm{P}\left(v_{\mu} \rightarrow v_{\mu}\right)$ and $\mathrm{P}\left(\bar{v}_{\mu} \rightarrow \bar{v}_{\mu}\right)$. In combination these measurements will be able to address the structure of the neutrino mass hierarchy and probe to new precision whether the mixing angle $\theta_{23}$ is non-maximal. If $\theta_{23}$ is non-maximal then the experiment will be sensitive to measuring the dominate coupling of the $v_{3}$ mass state to either the $v_{\mu}$ or $v_{\tau}$ flavor states. Through these measurements NOvA may also be able to provide some of the first information on the $\mathrm{CP}$ violating phase, $\delta_{C P}$, of the PMNS mixing matrix and provide tests of the 3-flavor mixing structure that PMNS style mixing assumes.

\section{MEASUREMENT TECHNIQUE}

The NOvA measurement technique for determining the neutrino mass hierarchy relies on the structure of the interference between the atmospheric and solar oscillation terms in the $v_{e}$ appearance probability $\mathrm{P}\left(v_{\mu} \rightarrow v_{e}\right)$. For neutrinos 


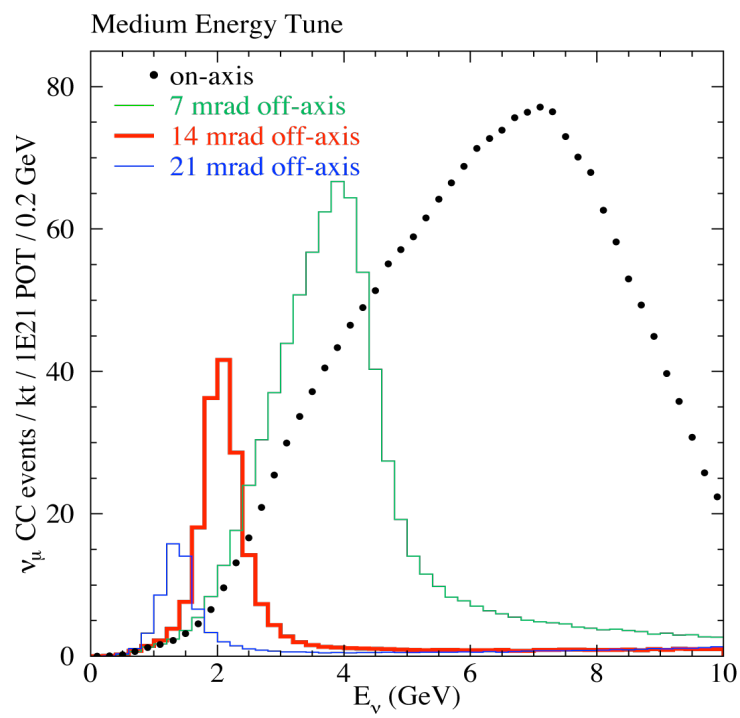

FIGURE 1. Un-oscillated neutrino energy spectrum at different angles off the primary beam axis. The NOvA experiment is sited at $14 \mathrm{mrad}$ to give a spectrum centered near $E_{v}=2 \mathrm{GeV}$.

and anti-neutrinos the transition probability can be written as,

$$
\begin{aligned}
P\left(v_{\mu} \rightarrow \stackrel{(-)}{v}_{e}\right) & \approx \sin ^{2} 2 \theta_{13} \sin ^{2} \theta_{23} \frac{\sin ^{2}(A-1) \Delta}{\left(A-1^{2}\right)} \\
& \stackrel{(+)}{-} 2 \alpha \sin \theta_{13} \sin \delta_{C P} \sin 2 \theta_{12} \times \sin 2 \theta_{23} \frac{\sin A \Delta}{A} \frac{\sin (A-1) \Delta}{A-1} \sin \Delta \\
& +2 \alpha \sin \theta_{13} \cos \delta_{C P} \sin 2 \theta_{12} \times \sin 2 \theta_{23} \frac{\sin A \Delta}{A} \frac{\sin (A-1) \Delta}{A-1} \cos \Delta
\end{aligned}
$$

Where,

$$
\alpha=\frac{\Delta m_{21}^{2}}{\Delta m_{31}^{2}}, \quad \Delta=\Delta m_{31}^{2} \frac{L}{4 E}, \quad A=\stackrel{(-)}{+} G_{f} N_{e} \frac{L}{\sqrt{2} \Delta}
$$

Unlike the reactor based $\bar{v}_{e}$ disappearance measurements which depend only on the $\theta_{13}$ angle, the long baseline appearance channel depends explicitly on the mixing angles $\theta_{13}, \theta_{23}$ and $\theta_{12}$, the phase $\delta_{C P}$ and the mass splitting $\Delta m_{31}^{2}$.

To see the sensitivity of $v_{e}$ appearance to the neutrino mass hierarchy, we examined appearance probabilities in the presence of matter. The dependancies can be rewritten in a more compact form in terms of the familiar atmospheric and solar oscillation terms as:

$$
\begin{aligned}
P\left(v_{\mu} \rightarrow v_{e}\right) & \simeq\left|\sqrt{P_{A t m}} e^{-i\left(\Delta_{32}+\delta\right)}+\sqrt{P_{\text {Sol }}}\right|^{2} \\
& =P_{\text {atm }}+P_{\text {sol }}+2 \sqrt{P_{\text {atm }} P_{\text {sol }}}\left(\cos \Delta_{32} \cos \delta_{C P} \mp \sin \Delta_{32} \sin \delta_{C P}\right)
\end{aligned}
$$

Where,

$$
\begin{aligned}
\sqrt{P_{\text {atm }}} & =\sin \theta_{23} \sin 2 \theta_{13} \frac{\sin \left(\Delta_{31}-a L\right)}{\Delta_{31}-a L} \Delta_{31} \\
\sqrt{P_{\text {sol }}} & =\cos \theta_{23} \sin 2 \theta_{12} \frac{\sin (a L)}{a L} \delta_{21} \\
a & =G_{F} N_{e} / \sqrt{2} \simeq 1 / 3500 \mathrm{~km}
\end{aligned}
$$




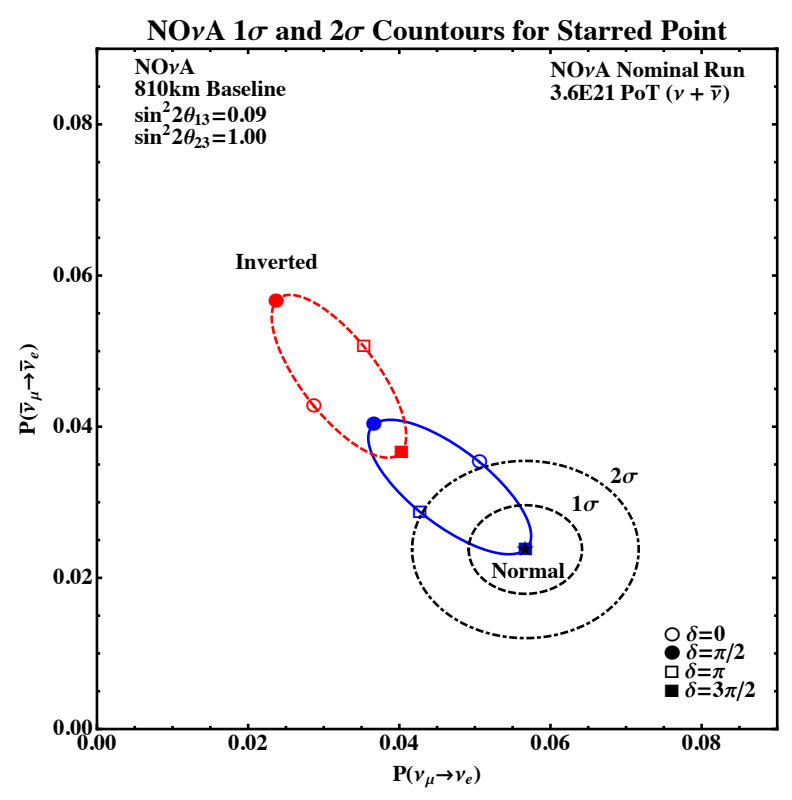

FIGURE 2. Bi-probability plot for the oscillation probabilities in the $v_{e}$ appearance channel for neutrinos and anti-neutrinos. The starred point represents a potential set of measurements for the NOvA experiment. The dashed contours represent the $1 \sigma$ and $2 \sigma$ statistical sensitivities at that point assuming a total exposure of $3.6 \mathrm{E} 21$ protons on target.

Here the relative sign between the cosine and sine terms in the interference component of (3) depends on whether we are observing the neutrino or anti-neutrino oscillation channels. If the $\mathrm{CP}$ violating phase delta is non-zero this leads to an enhancement/suppression of the neutrino/anti-neutrino transition probabilities relative to each other. Similarly the dependence of $\sqrt{P_{a t m}}$ in (4) on $\Delta_{31}$ leads to a separate enhancement/suppression effect which is dependent on the relative sign of the mass splitting between $v_{3}$ and $v_{1}$. This causes the $v_{e}$ appearance transition process to be sensitive to the neutrino mass hierarchy through a comparison of the rates for the neutrino and anti-neutrino channels. The matter effect scales with $a$ times the baseline over which the neutrinos travel. For a long baseline like the $810 \mathrm{~km}$ long neutrino flight path of NOvA, the size of the effect is proportional to $a L=0.23$. For a shorter baseline like the $295 \mathrm{~km}$ one for T2K the size of the effect is only $a L=0.08$.

The oscillations probabilities, $\mathrm{P}\left(v_{\mu} \rightarrow v_{e}\right)$ and $\mathrm{P}\left(\bar{v}_{\mu} \rightarrow \bar{v}_{e}\right)$ can be plotted against each other, parametrically in the phase $\delta_{C P}$ for the two possible signs of $\Delta m_{31}^{2}$. When this is done, two separate ellipses emerge corresponding to the normal and inverted neutrino mass hierarchies. This is shown in Fig. 2 where the ellipses correspond to the NOvA baseline and neutrino energy. Experimentally performing the measurements of $\mathrm{P}\left(v_{\mu} \rightarrow v_{e}\right)$ and $\mathrm{P}\left(\bar{v}_{\mu} \rightarrow \bar{v}_{e}\right)$ then givens a point in this space that should lay on the theoretic curves. In the example of Fig. 2, observation of the probabilities at the starred point, with the NOvA statistically sensitivities corresponding to 3.6E21 protons on target, would lead to the establishment of the normal mass hierarchy at the $95 \% \mathrm{CL}$, and provide evidence for a non-zero phase $\delta_{C P}$.

NOvA's sensitivity to the resolution of the neutrino mass hierarchy can be examined over the full range of $\delta_{C P}$. For the nominal exposure of 3.6E21 protons on target, split between neutrino and anti-neutrino running and for a maximal $\theta_{23}$, the NOvA experiment would be sensitivity to resolving the mass hierarchy at the $95 \% \mathrm{CL}$ over a third of the values of $\delta_{C P}$ as shown in Fig. 3. Combining the NOvA sensitivities with those of T2K, breaks the degeneracies in the overlap regions between the ellipses of Fig.2 and leads to $1 \sigma$ sensitivities over all values of $\delta_{C P}$.

For the $v_{\mu}$ disappearance measurement, the sensitivity of the experiment to measuring $\theta_{23}$ corresponds to ability to measure the position of the dip in the oscillated neutrino energy spectrum as shown in Fig. 4. This sensitivity is driven by the energy resolution that can be obtains in the reconstruction of $v_{\mu}$ charged current interactions. Based on current detector performance and reconstruction techniques, the NOvA experiment is able to achieve a $4.5 \%$ energy resolution for quasi-elastic $v_{\mu} \mathrm{CC}$ events and is able to achieve a $6 \%$ energy resolution for non-QE events. These energy resolutions have been combined with cosmic ray induced background rejection efficiencies, determined from the far detector data, to determine the sensitivities of the NOvA experiment to measurement of $\theta_{23}$. Figure 6 shows these sensitivities for both maximal and non-maximal values of $\theta_{23}$. The updated sensitivities show that for the baseline exposure of 3.6E21 POT, NOvA will improve on the current best fit values for any measured value of $\theta_{23}$. 


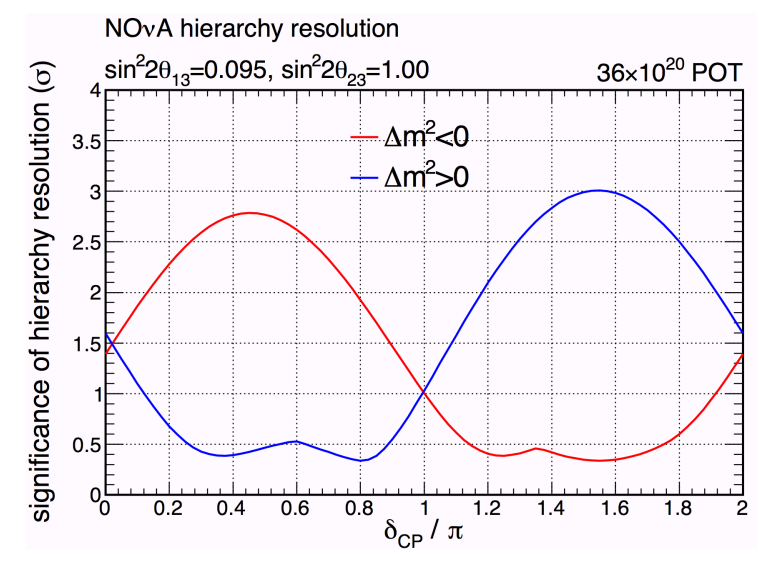

FIGURE 3. Statistical sensitivity of the NOvA experiment to the resolution of the neutrino mass hierarchy as a function of the phase $\delta_{C P}$.

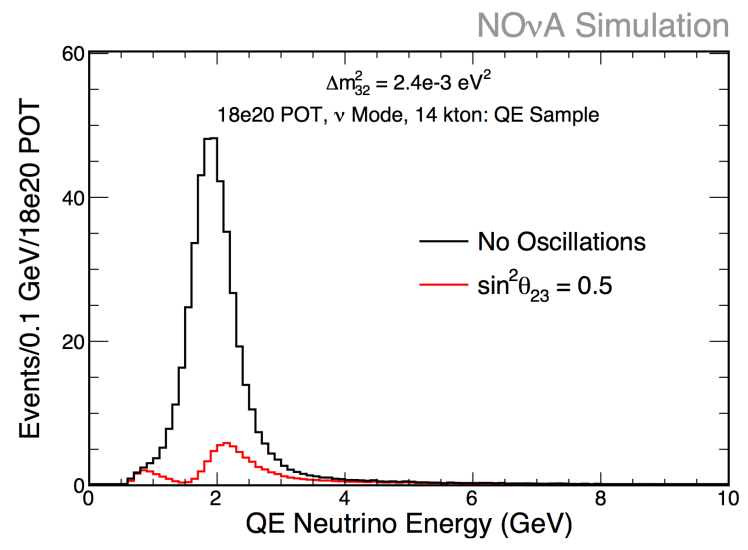

FIGURE 4. Simulated $v_{\mu}$ energy spectrum for the reconstruction of $v_{\mu}$ quasi-elastic charged current interaction.

If NOvA observes a measurement in the $v_{\mu}$ disappearance channel that is consistent with a non-maximal $\theta_{23}$, then the dominate coupling of the third neutrino mass state $\left(v_{3}\right)$ can be determine through the corresponding measurements of the $v_{e}$ appearance channels in much the same manner as the determination of the mass hierarchy.

In this case the term of (1) which is proportional to $\sin 2 \theta_{23}$ is enhanced if $\theta_{23}>45^{\circ}$ indicating a dominate coupling to the $v_{\mu}$ flavor state or suppressed if $\theta_{23}<45^{\circ}$ indicating a dominate coupling to the $v_{\tau}$ flavor state. The effect of these enhancements or suppressions are to further bifurcate the bi-probability ellipses into an upper and lower set of solutions as shown in Fig. 5. For the nominal NOvA beam exposure this leads to a greater than 95\% CL determination of the octant to which $\theta_{23}$ couples over all values of $\delta_{C P}$ for $\sin ^{2} 2 \theta_{23}=0.97$ in either of the dominate coupling solutions.

\section{DETECTOR RESPONSE}

The NOvA detection cells are designed as $15.6 \mathrm{~m}$ long tubes with $3.9 \times 6.6 \mathrm{~cm}$ rectangular cross sections. They are made by extruding highly reflective polyvinyl chloride (PVC) into thin walled modules which are each 16 cells wide as shown in Fig. 7. Each cell is filled with a mineral oil based liquid scintillator and strung with a single wave-shifting fiber which is looped down the cell and whose ends are readout by an avalanche photo diode (APD). The modules are assembled into $15.6 \times 15.6 \mathrm{~m}$ cross section detector planes. 896 detector planes were then arranged in alternating $\mathrm{X}$ measuring and $\mathrm{Y}$ measure orientations to form the full $14 \mathrm{kt}$ range stack/calorimeter design that is the NOvA far detector. A minimum ionizing particle crossing the cell horizontally across the $6.6 \mathrm{~cm}$ direction results in an average 


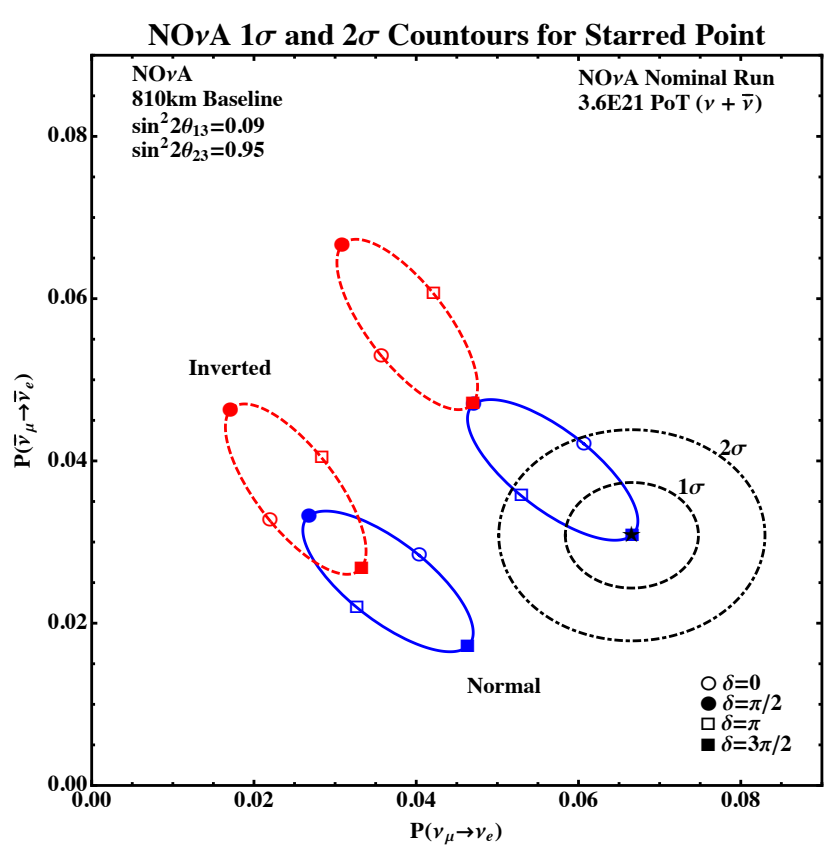

FIGURE 5. Bi-probability ellipses for the $v_{e}$ appearance channels with a non-maximal value of $\theta_{23}$.

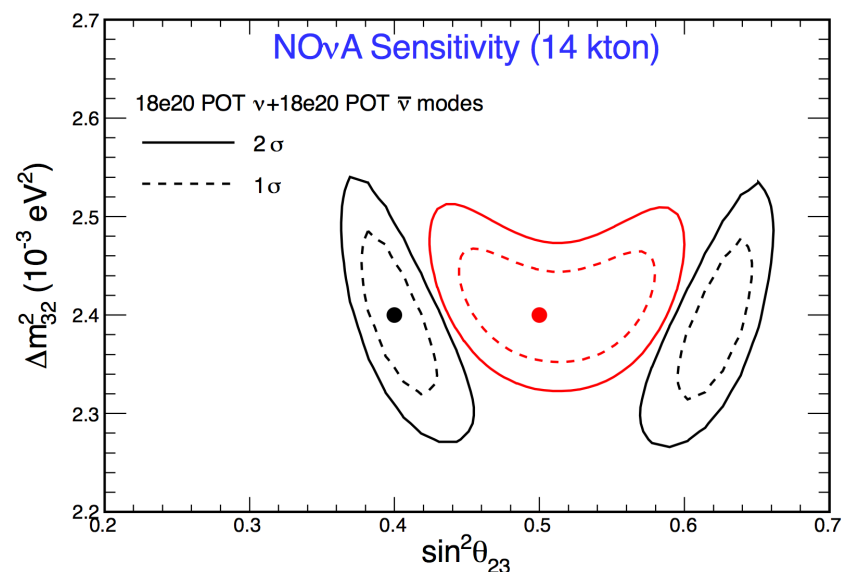

FIGURE 6. NOvA sensitivity contours for maximal and non-maximal values of $\theta_{23}$.

$\mathrm{dE} / \mathrm{dx} \approx 12.9 \mathrm{MeV}$. To achieve a signal to noise ratio of $10: 1$, the light output transmitted to the APD readout for a particle passing through the far end of the cell needs to be 30-38 photo electrons.

The measured single cell response for muons passing through the far end of the detector cells has been measured and is shown in Fig. 8. The measurements show that the NOvA detector cells in both the horizontal and vertical orientations are able to meet the technical design report goal. These measurements show that all of the individual components, the custom liquid scintillator, the more than 30 meters of wave-shifting fiber in each cell, the reflectivity of the PVC, the amplifier gains and noise spectra of the electronics are all at or above the level they needed to be for the detector to achieve its design parameters.

The single hit efficiency as a function of the position along the cell through which the particles pass has also been measured in the far detector and is shown in Fig. 9. The detector is able to achieve a better than 0.9 efficiency across the full length of the $15.6 \mathrm{~m}$ detections cells and close to 0.99 in the upper half of the detection cells near the APDs readouts. The resulting efficiency for the detection and reconstruction of tracks corresponding to the passage of minimum ionizing particles through the detector is greater than 0.99 over the full fiducial volume of the detector. 
High reflectivity PVC extrusion (16 cells/extrusion)

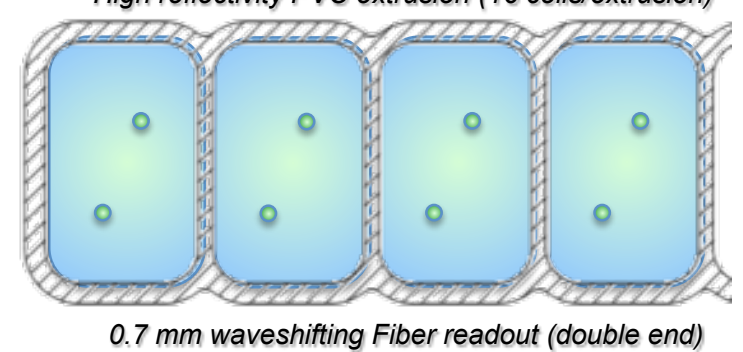

FIGURE 7. NOvA readout cell geometry. Cells are extruded in groups of 16 to form a thin walled PVC module. Each cell is strung with a wavelength shifting fiber and filled with liquid scintillator.

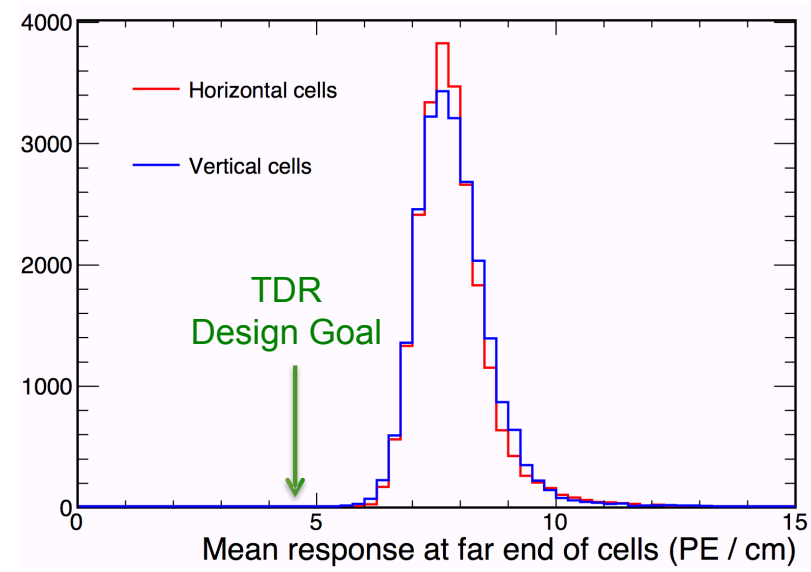

FIGURE 8. NOvA far detector readout cell single hit response curve.

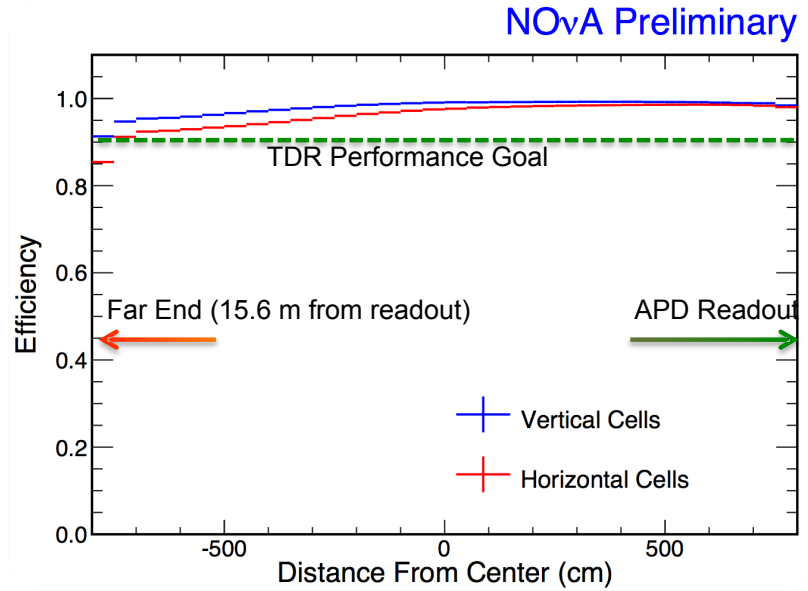

FIGURE 9. Readout cell efficiency vs hit position along the cell for the NOvA far detector. The technical design report (TDR) performance goal is set at 0.90 . 


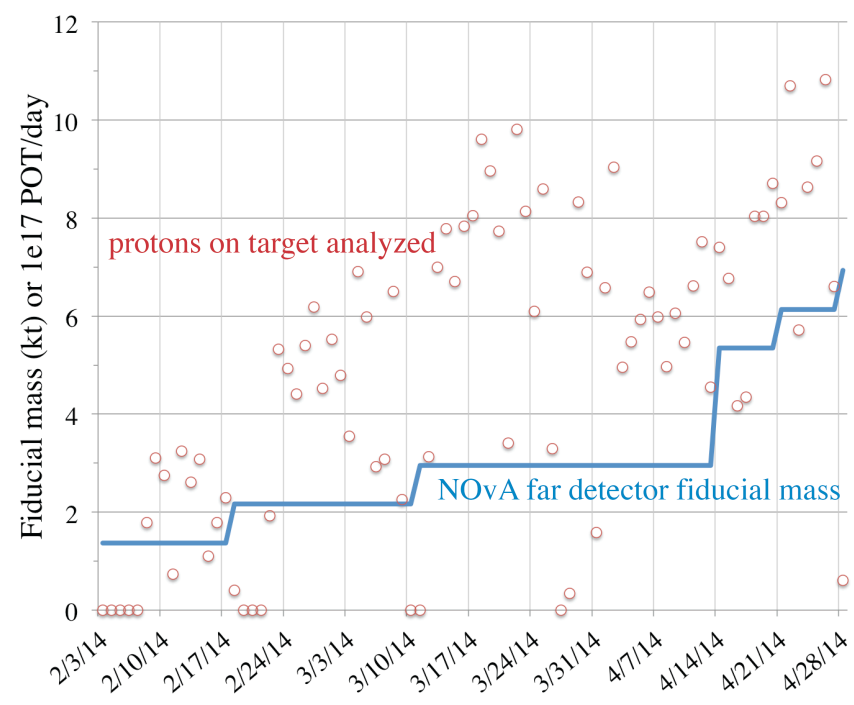

FIGURE 10. NOvA far detector fiducial mass used for physics data taking as a function of time.

\section{DETECTOR OPERATIONS}

The NOvA detector began commissioning and operations of the first kiloton of fully instrumented detector mass in August of 2013. The first early physics operations started on Feb 3, 2014. During the period from February through July 2014 the remaining $13 \mathrm{kt}$ of detector mass were constructed, instrumented, commissioned and then added to the physics data taking. Figure 10 shows the beam exposure that was received and the growth of the fiducial mass of the far detector as new blocks were commissioned and added to the readout. During this period the physics data taking maintained an average uptime of $>85 \%$.

The volume of data that was integrated during the first four months of operations (Feb-May 2014) was approximately $1.7 \%$ of a nominal year at full detector mass and full design beam power.

\section{OBSERVATION OF FIRST $v$ EVENTS}

The interaction and observation of the first NuMI induced neutrino interaction in the NOvA far detector occurred on Nov. 12, 2013. The first event shown in Fig. 11 shows the XZ and YZ view projections of the raw hit information along with higher level reconstructed quantities. The event exhibits a defined and isolated interaction vertex inside the fiducial boundaries of the detector along with the resulting energy deposition and reconstructed trajectories of three charged particles. The displacement between the primary vertex and the start of the third charged track is characteristic of the event signature for the conversion of a neutral particle in the detector and can be used to distinguish neutral current events with an associated production of a $\pi^{0}$ from $v_{e}$ events.

Similarly Fig. 12 shows a $v_{\mu}$ charge current candidate that was identified during the early physics running. The event demonstrates the strengths of the NOvA detector design and the extremely quiet nature of the actual operational detector. The pictured event demonstrates the well defined interaction point formed by the intersection of the long narrow track like object [the muon] and the more diffuse shower-like plume which is easily identified as hadronic material from the nuclear recoil. The muon itself is composed of a total of 100 individual hits, 51 in the XZ-view and 49 in the YZ-view. The muon stretches over a length of more than $7 \mathrm{~m}$ and while the individual projects of the track have individual missing planes or "gaps" owing to either the track passing through inactive material or low efficiency cells in the detector, the full trajectory is easily reconstructable both by eye and algorithmically. The energy of the muon is easily determined by it's trajectory and range in the detector. The nuclear part of the interaction can similarly be reconstructed due the fine segmentation of the detector and the total energy can be determined through the calorimetric sum of visible energy in the detector. The noise in the detector, both accidental and from the cosmic ray flux that the detector experiences being on the surface, has minimal effect on the event detection and reconstruction 


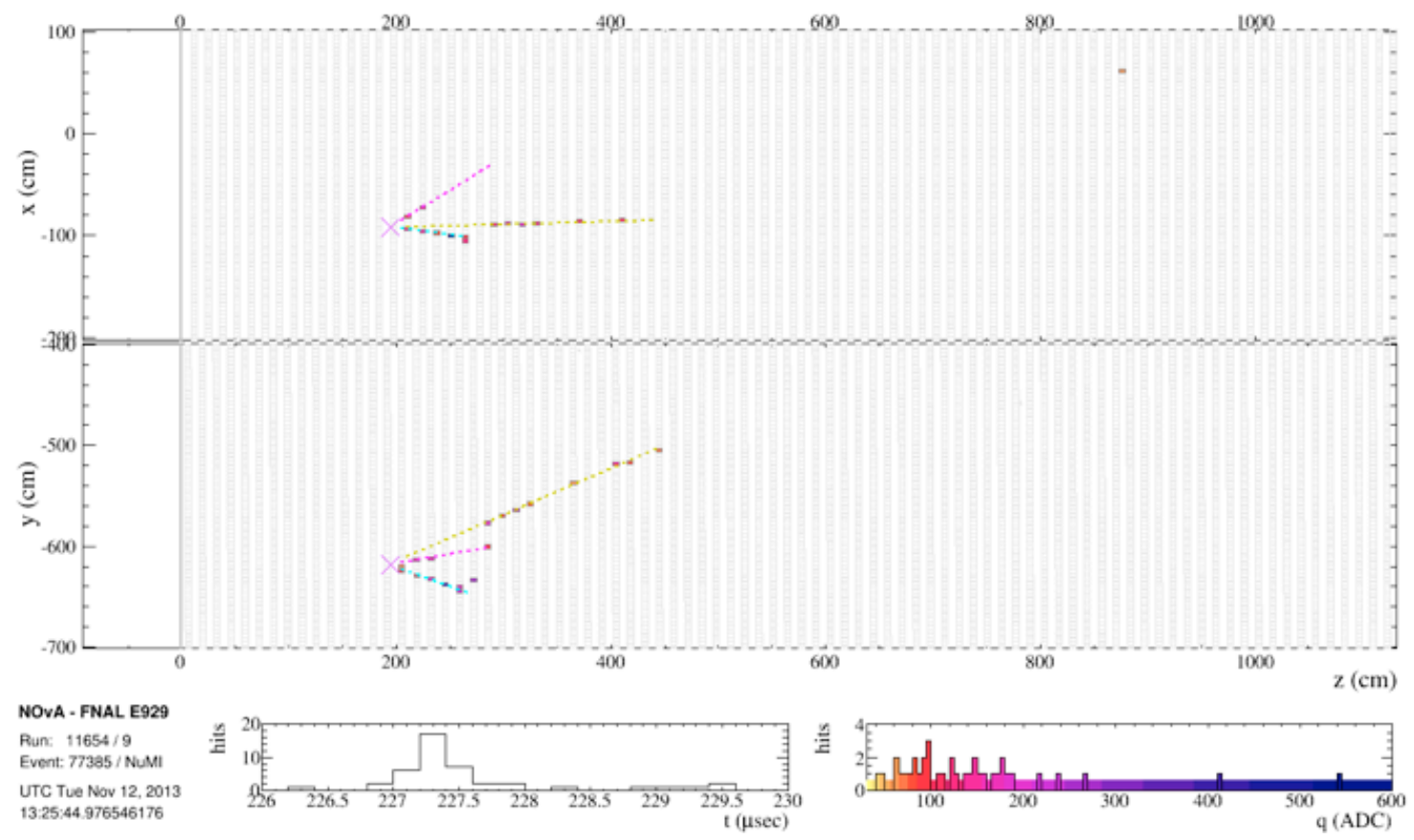

FIGURE 11. First observed neutrino candidate in the NOvA far detector.

due to the low singles rates for individual channels, the timing resolution of the hits and the fine granularity and size of the detector which reduces the effect of accidentals and pileup.

Due to the extremely low expected rate of neutrino interaction in the far detector during initial commissioning ${ }^{1}$, the observation of neutrino candidates were combined with detailed checks of the GPS based timing and synchronization system to determine if the event candidates were indeed induced by the beam flux from the NuMI neutrino beam. Apriori calculations were performed to predict the time offset at which the neutrino beam would cross the far detector relative to the time $t_{0}$ at which the NuMI accelerator spill signal was received by the NOvA master timing system located at Fermilab. The plot in Fig. 13 shows the activity in one of the NOvA near detectors ${ }^{2}$ and the neutrino candidates events as a function of the time in microseconds from the reception of the trigger signal $t_{0}$. The far detector data have a time offset of $2700.56 \mu s$ subtracted from their events times, corresponding to the calculated flight time between the two detectors. The distributions show a clear event excess over the predicted $10 \mu s$ time window corresponding to the NuMI beam. A similar rate excess was observed in the distribution of "rock muon" events $^{3}$ which aligned with the known beam direction.

Timing and validation of the NOvA near detector was performed by looking for a simple rate excess in the raw hit multiplicities. Due the much higher neutrino induced activity rates in the near detector, the beam peak as shown in Fig. 14 was observed after 13.38 seconds of total detector live time and with only $12 \%$ of the detector electronics in the actual readout.

\section{FAR DETECTOR DATA ANALYSIS}

The data collected with the NOvA far detector over the first twelve months of commissioning and operations has been used to extensively validate the simulation chain and to tune the modeling of the detector and its readout in the simulations. The validation was performed using a high statistics sample of minimum bias data. The minimum bias

\footnotetext{
${ }^{1}$ First observations were performed with 1-2 kt of detector mass and a average beam power of $280 \mathrm{kw}$

2 Near detector on the surface, located $112 \mathrm{mrad}$ off-axis

$3 v_{\mu}$-CC events occurring in the rock surrounding the detector
} 


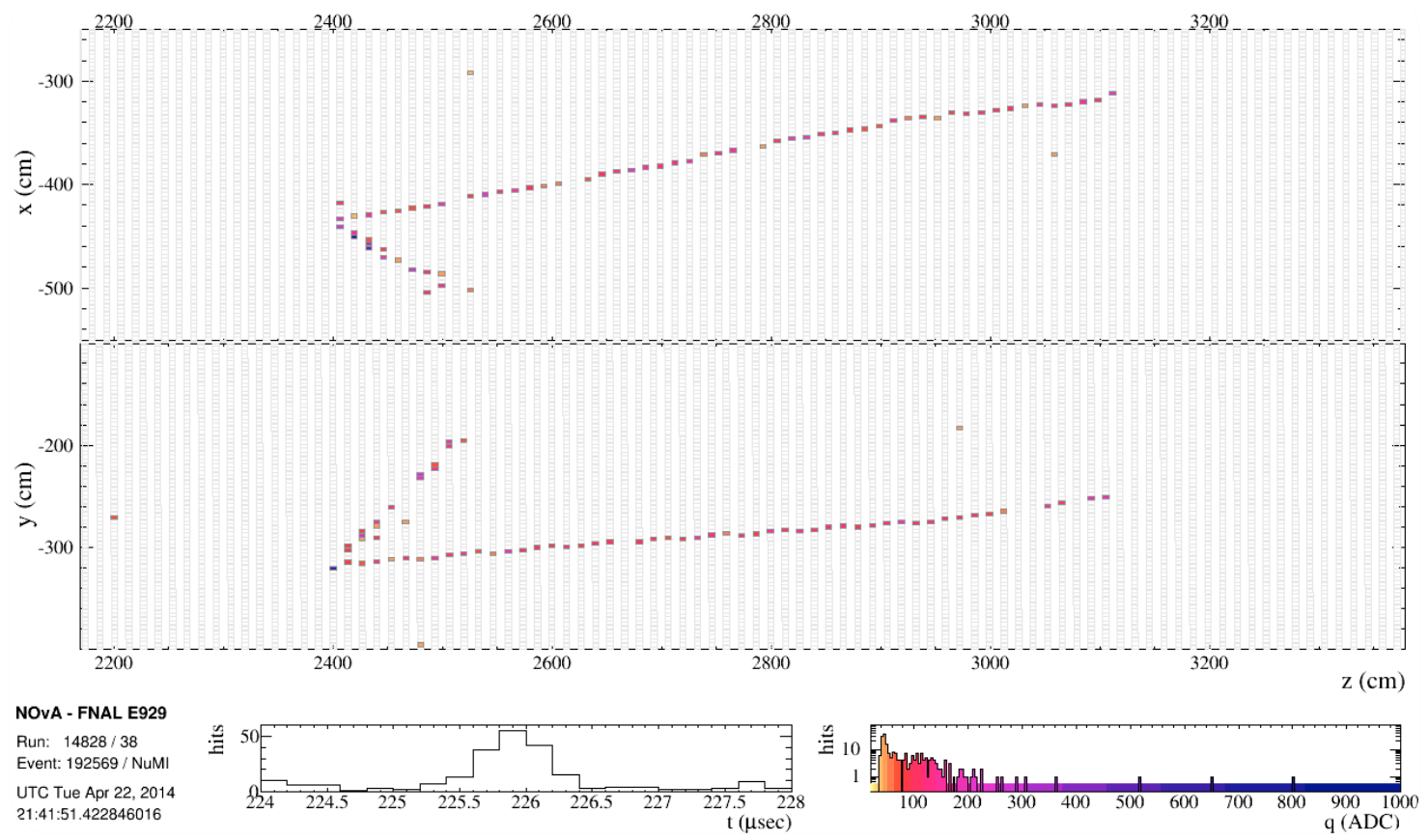

FIGURE 12. $v_{\mu}$-CC candidate interaction NOv A far detector.

NOvA Preliminary

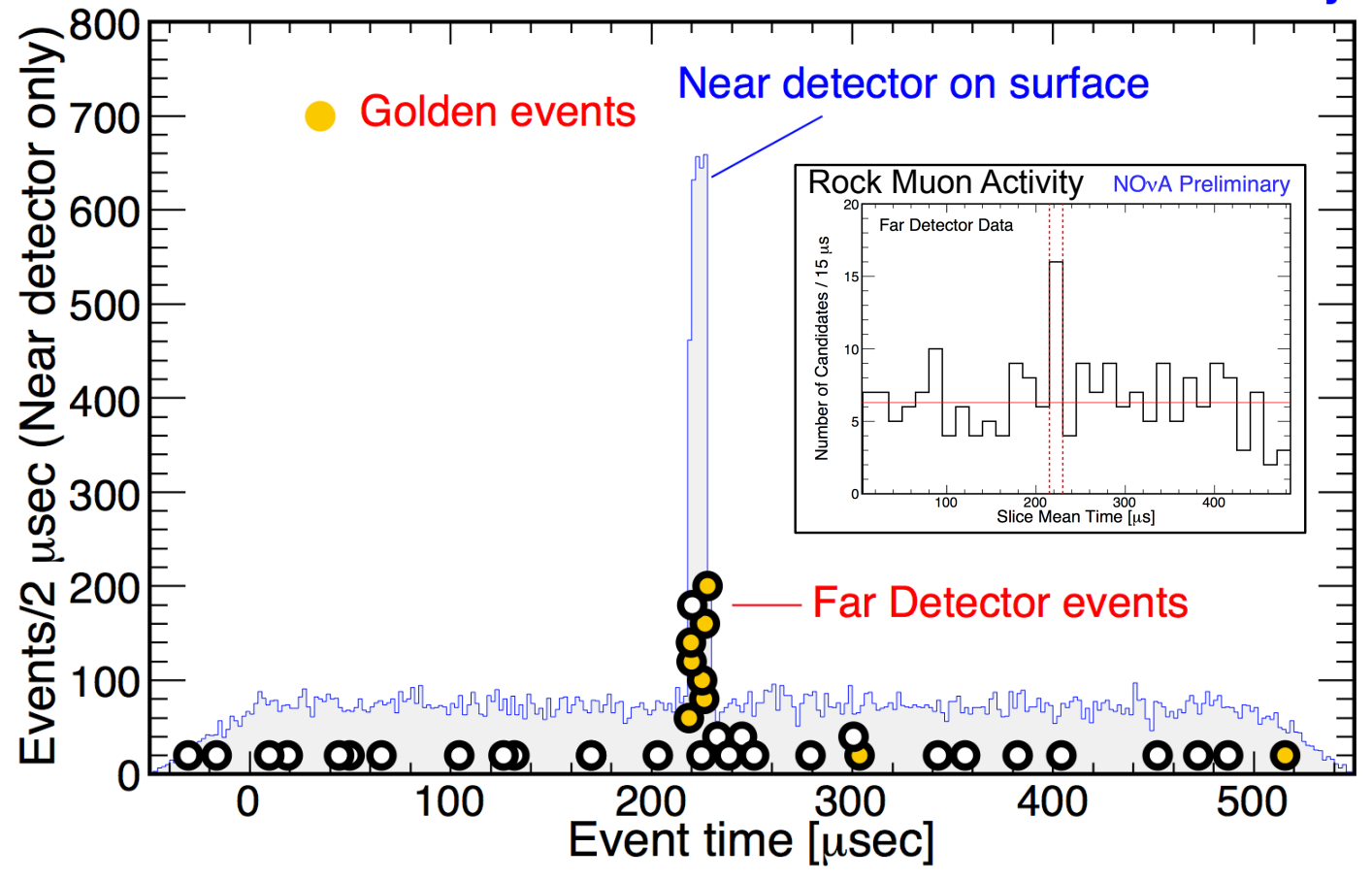

FIGURE 13. Candidate neutrino events observed in the NOvA far detector as a function of the time difference $(\Delta t)$ in $\mu$ s between the reconstructed event and the reception of the accelerator spill trigger. The distribution is superimposed on the distribution of hit times from the NOvA near (surface) detector. Far detector time offsets are corrected for measured data acquisition system timing delays and time of flight between NuMI target station and NOvA far detector. 


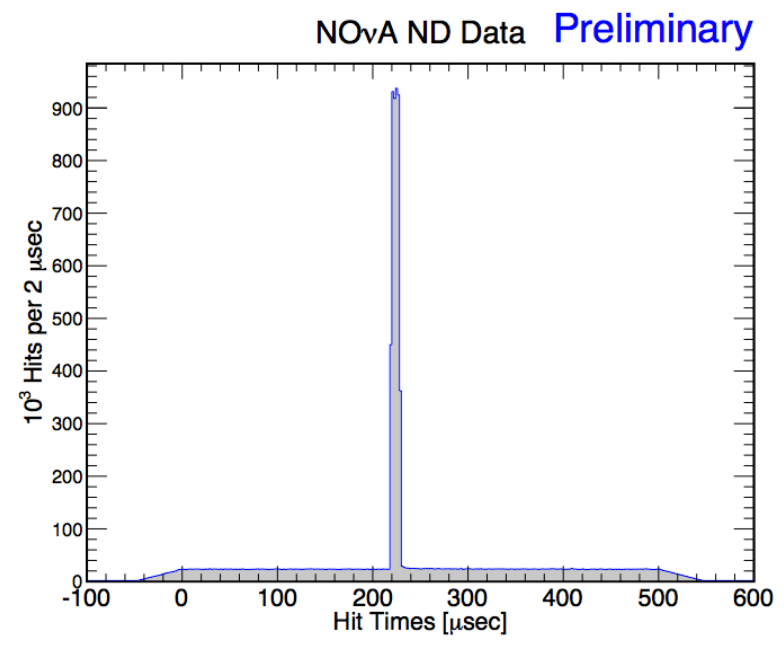

FIGURE 14. NOvA near detector hit multiplicities as a function of time since the reception of the trigger $t_{0}$. The rate excess from 218-228 $\mu$ s corresponds to the NuMI beam.

data was collected using $550 \mu s$ long time windows which were triggered with a periodic (software based) pulser and were uncorrelated to the neutrino beam. As similar set of $550 \mu \mathrm{s}$ readout windows were triggered on the accelerator trigger signals and centered on the $10 \mu s$ NuMI beam spills. This samples provided leading and trailing side bands for pre and post spill conditions studies. The periodic pulser for the readout varied during the commission phase of the detector from $10-40 \mathrm{~Hz}$ in order to collect a data sample that represented an exposure that was more than $100 \times$ that of the integrated live time represented by the sum of the $10 \mu \mathrm{s}$ beam spill windows.

Because the NOvA far detector is situated on the surface with only a $3 \mathrm{~m}$ earth equivalent overburden composed of concret and barite, each $550 \mu s$ far detector readout time window contains an average of 6.6 cosmic ray muons per kiloton of detector mass. This muon rate has allowed the minimum bias sample that was collected during detector commissioning and early physics running to sample both the full phase space of the cosmic ray spectrum and the full geometric extent of the detector with high statistics.

The comparison of data to Monte Carlo simulation in Fig. 15 shows the position of cosmic ray induced vertices that were identified by the NOvA reconstruction software. The data and Monte Carlo agree to high precision across the full range of the detector's fiducial volume and only show minor disagreement for events with reconstructions that are projected outside of the detector's physical extent. Similarly Fig. 16 shows the angle of the reconstructed muons with respect to the NuMI beam direction. The distributions agree over the full range of track angles that are reconstructable by the rectangular geometry of the detector. Deviations occur for tracks that cross nearly parallel to the $\mathrm{XZ}$ or YZ measuring planes where there is a loss of information that prohibits 3-dimensional reconstruction. These tracks are perpendicular to the beam/detector axis and are removed by the first level of selection cuts when performing the neutrino analyses.

The data to Monte Carlo comparison shown in Fig. 17 is the output classifier from the electron identification neural network when run over cosmic ray induced activity. The distribution shows the large "background-like" peak corresponding to cosmic ray activity near output values of 0 and " $v_{e}$-interaction-like" activity near output values of 1. The distribution shows excellent agreement between the simulation and the actual data across the full range of classifications.

\section{$v_{e}$ Selection}

The selection of $v_{e}$ charge current events in the NOvA far detector is based on two complimentary particle identification approaches. The first method of electron-ID (EID) uses a neural network classification scheme based on the electromagnetic shower profile variables calculated by a series of clustering, vertexing and shower reconstruction algorithms. The output of the EID process is shown in Fig. 18 for the Monte Carlo based predictions for the oscillated $v_{\mu} \rightarrow v_{e}$ flux as well as for backgrounds attributed to neutral current interactions, mis-id'd $v_{\mu}$ interactions, and the 


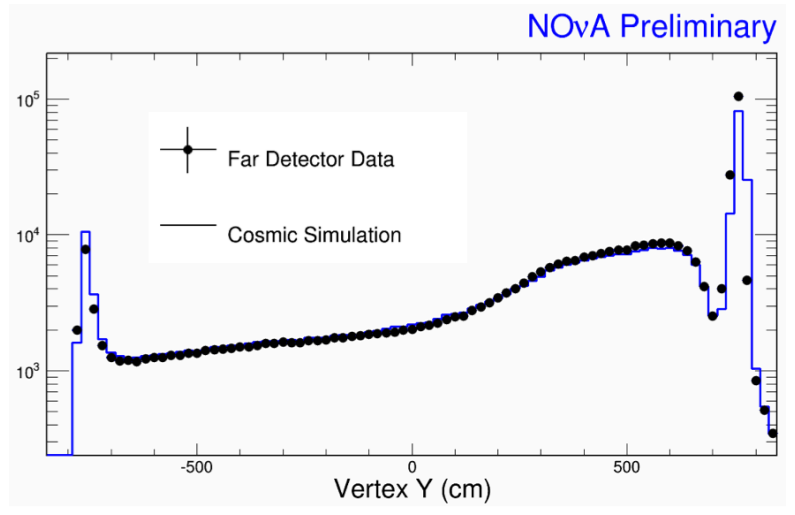

FIGURE 15. Far detector data to Monte Carlo comparisons for cosmic ray spectra after full reconstruction. Distribution shows the vertical position of reconstructed vertices across the detector. Physical extent of the detector ranges from $-784 \mathrm{~cm}$ (bottom) to $784 \mathrm{~cm}$ (top).

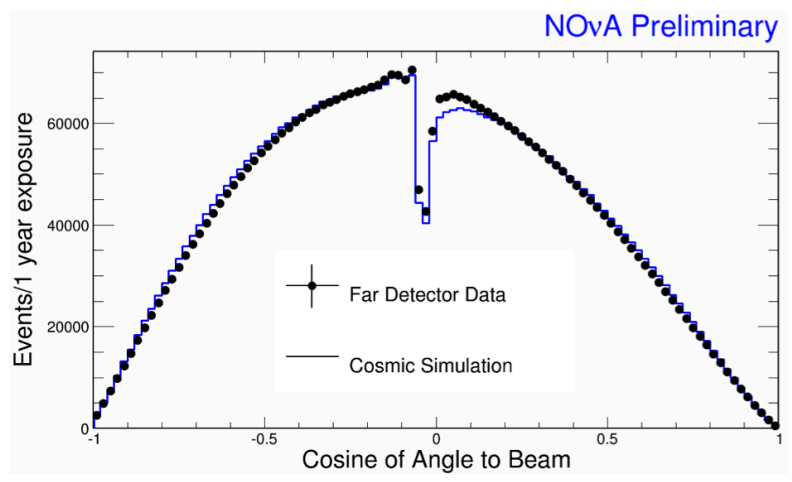

FIGURE 16. Far detector data to Monte Carlo comparison of the reconstructed angle of the cosmic rays and the direction of the NuMI neutrino beam.

intrinsic $v_{e}$ content of the NuMI neutrino beam. These distributions are scaled to a nominal $1 \mathrm{yr}$ exposure of $6 \times 10^{20}$ POT at full detector mass and design beam power. Overlaid on this is the real cosmic ray induced activity from the far detector. The cosmic ray background distribution is scaled down the to represent $120 \mathrm{~s}$ of detector readout, corresponding to the effective live time integrated by the beam spills to achieve an exposure of $6 \times 10^{20} \mathrm{POT}$.

The second method of $v_{e}$ event identification uses a template library matching scheme between the real events and a large Monte Carlo generated library of templates. This method uses the hit level data instead of the higher level reconstruction objects to select the best matches to the candidate event from a library of 77 million events representing both signal and background interactions. The library template method achieves similar performance, as measured by a Signal $/ \sqrt{\mathrm{Bkg}}$ figure of merit, to the neural network. However because the library template method is not reliant on the reconstruction chain is able to serve as an independent check to the EID approach. Together they can be used to study the systematic uncertainties in both approaches.

Cosmic rays are further rejected from the analysis sample through the use of simple cuts on three selection variables. The selection criteria examines the ratio of the transverse to total moment of the event, $P_{t} / P$ and then cuts on it to force the directionality of showers to align with the beam direction. The second selection cut that is used is performed on the maximum position in $\mathrm{Y}$ of hits in the event. The cut removes particles that are entering the detector from the top and eliminates cosmic ray induced activity, primarily neutrons and gammas, that leak through or originate from interactions in the overburden. The third selection criteria that is used to reject cosmic ray induced events, is a cut on the minimum gap between the reconstructed vertex position and the hit activity of the outgoing particles. This cut removes events with poorly reconstructed vertices or where other portions of the reconstruction were not efficient.

After the application of all the electron identification and selection criteria, the projected signal and background rates were calculated. These rates are shown in Table 1 broken down by selection criteria. The signal estimates shown 


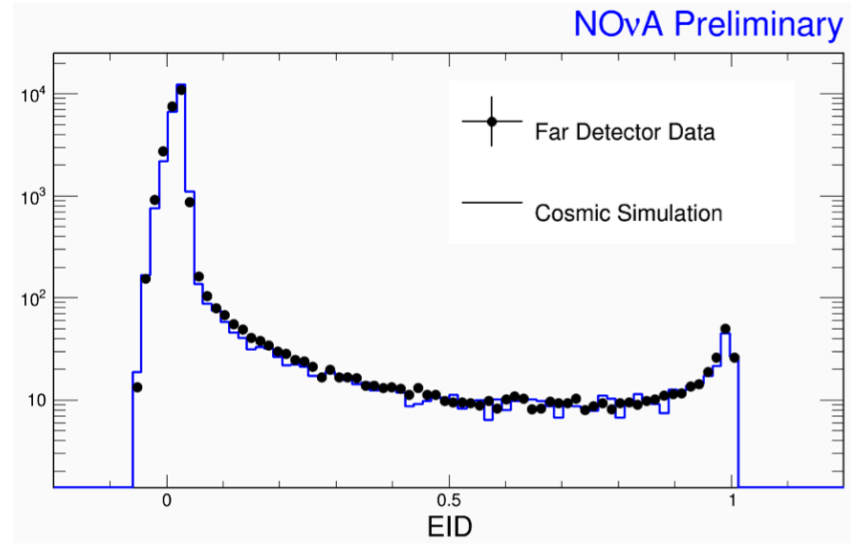

FIGURE 17. Data to Monte Carlo comparison of cosmic ray backgrounds subjected to electron ID neural network selection criteria.

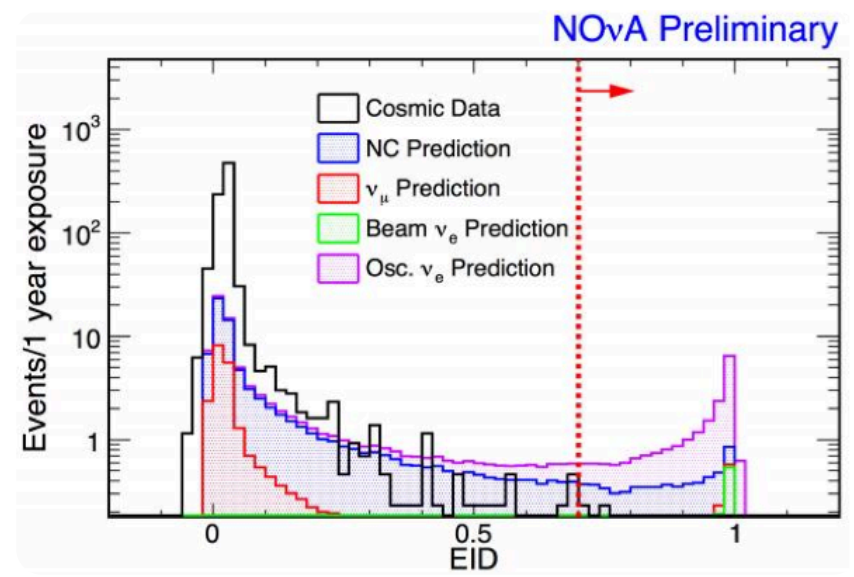

FIGURE 18. $v_{e}$ selection through Electron ID (EID) neural network. Cosmic ray data from the far detector has been normalized to 1 year of nominal exposure (120 s live readout) induces less than 0.5 events in the signal region.

in this table are calculated to leading order. They include only simple oscillations for a maximal $\theta_{23}$ and do not include matter effects. They are averaged over mass hierarchies and values of $\delta_{C P}$. The rates correspond to a nominal one year exposure of $6 \times 10^{20} \mathrm{POT}$ at full detector mass and design beam power.

The data driven cosmic ray background estimates demonstrate a 40 million to 1 cosmic ray rejection rate, yielding a an expected background of 0.5 on a signal of $14 v_{e}$ events.

\section{$v_{\mu}$ Selection}

The selection of $v_{\mu}$ charged current events in the NOvA far detector is similar to that of the $v_{e}$ selection but uses a boosted decision tree (BDT) approach to separate out the neutrino interactions from the cosmic ray backgrounds. The same data driven approach to determining the cosmic ray induced background was used. The full distribution for the total $v_{\mu}$ classifier is shown in Fig. 19. The $v_{\mu}$ analysis puts a cut on the BDT and then applies the remaining selection cuts based on the fiducial containment of the events. This process achieves a cosmic ray rejection rate of 20 million to 1 , resulting in a prediction of one cosmic ray background event per nominal year of running.

The full breakdown of expected signal and background rates for the first year of nominal running are shown in Table 2 while Fig. 20 shows the the expected $v_{\mu}$ survival spectrum as a function of energy and compared to the extrapolated but unoscillated near detector spectrum. 
TABLE 1. Signal and background estimates for $v_{e}$ selection for an exposure of $6 \times 10^{20}$ protons on target. Estimates assume the full $14 \mathrm{kt}$ detector. Cosmic ray induced backgrounds are determined from real data taken with the detector under nominal running conductions.

\begin{tabular}{lllllcc}
\hline & $v_{e}$ Signal $^{*}$ & NC & Beam $v_{e}$ & $v_{\mu} \mathbf{C C}$ & Cosmic Ray $^{\dagger}$ & All Background \\
\hline All Events & 36.7 & 380 & 28.1 & 557 & $19 \mathrm{M}$ & $19 \mathrm{M}$ \\
\hline Pre-selection & 24.7 & 83.5 & 2.9 & 30.0 & $56 \mathrm{k}$ & $56 \mathrm{k}$ \\
Vertex Gap & 24.6 & 81.8 & 2.9 & 29.6 & $55 \mathrm{k}$ & $55 \mathrm{k}$ \\
$P_{T} / P$ & 22.0 & 59.6 & 2.6 & 24.3 & 1248 & 1334 \\
Maximum Y & 21.2 & 57.4 & 2.5 & 23.0 & 834 & 917 \\
\hline Neural Net & $\mathbf{1 3 . 9}$ & 3.9 & 1.5 & 0.7 & 0.5 & $\mathbf{6 . 5}$ \\
Library Template & $\mathbf{1 4 . 0}$ & 3.5 & 1.5 & 1.1 & 0.9 & $\mathbf{7 . 0}$ \\
\hline
\end{tabular}

${ }^{*}$ Leading order, simple oscillations without matter effects, averaged over $\delta_{C P}$

$\dagger$ data driven

TABLE 2. Signal and background estimates for $v_{\mu}$ selection for an exposure of $6 \times 10^{20}$ protons on target. Cosmic ray induced backgrounds are determined from real data taken with the detector under nominal running conductions.

\begin{tabular}{lllllcc}
\hline & Osc. $v_{\mu}$ & NC Bkg & Osc. $v_{e}$ & Beam $v_{e}$ & Cosmic Bkg* & Total Bkg \\
\hline All Events & 127 & 380 & 37 & 10 & $19 \mathrm{M}$ & $19 \mathrm{M}$ \\
\hline Cosmic Veto & 125 & 273 & 36 & 10.0 & $6 \mathrm{M}$ & $6 \mathrm{M}$ \\
Fiducial Containment & 109 & 195 & 28 & 7.5 & $120 \mathrm{k}$ & $120 \mathrm{k}$ \\
\hline$v_{\mu}$ CC ID & 86 & 5 & 0.4 & 0.2 & $44 \mathrm{k}$ & $44 \mathrm{k}$ \\
Cosmic Reject & $\mathbf{7 5}$ & 4 & 0.3 & 0.1 & 1 & $\mathbf{5 . 4}$ \\
\hline
\end{tabular}

* data driven

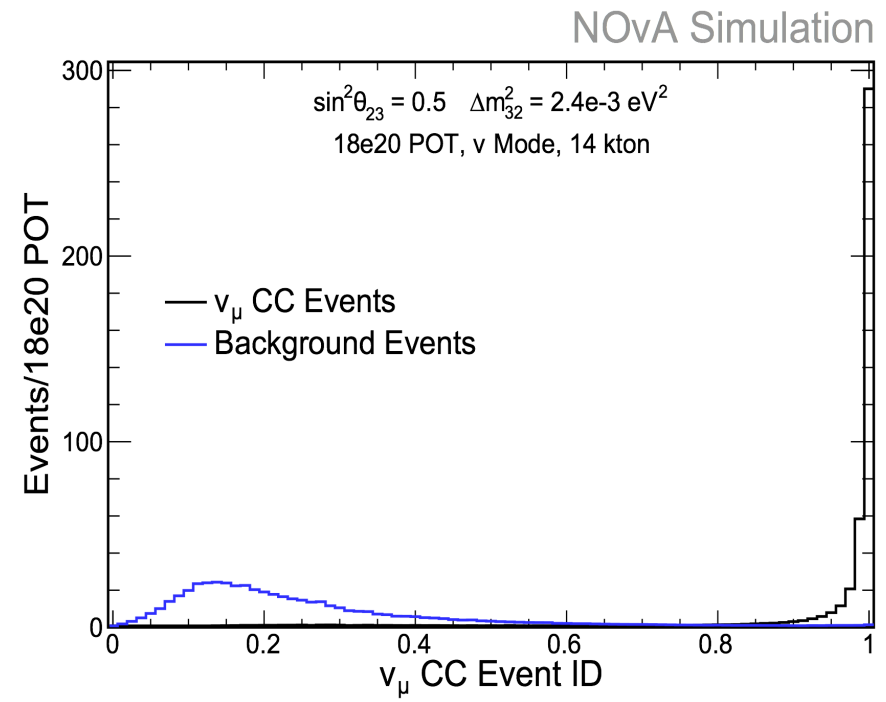

FIGURE 19. Cosmic ray induced background rejection under $v_{\mu}$ selection criteria. 


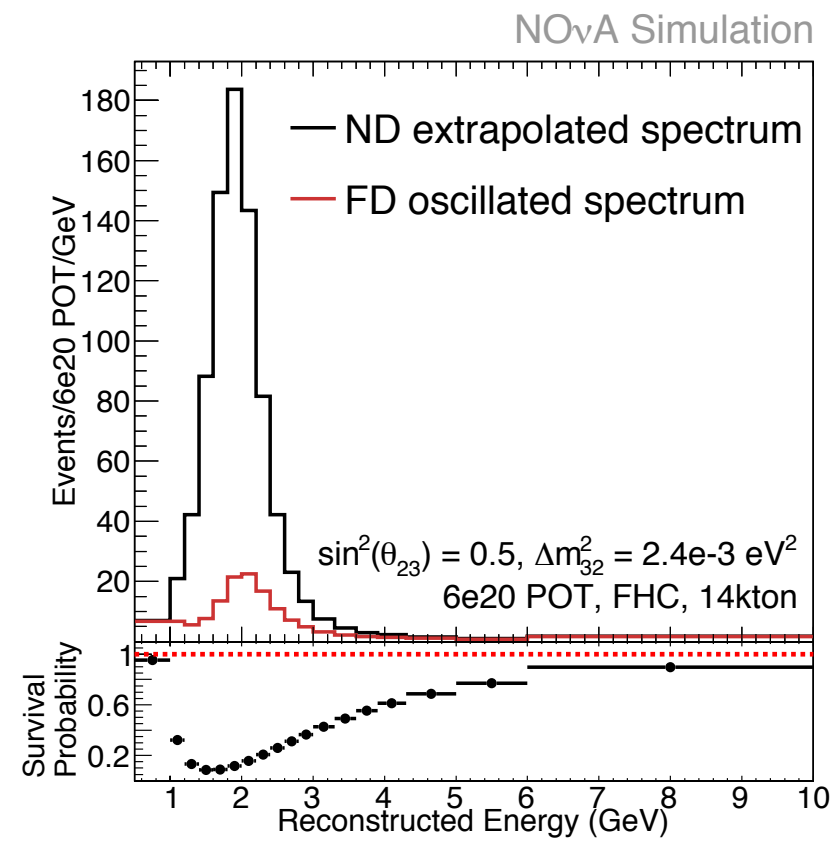

FIGURE 20. Oscillated and un-oscillated $v_{\mu}$ fluxes extrapolated to the NOvA far detector as a function of energy. Flux is normalized to a nominal 1 year exposure of exposure of $6 \times 10^{20} \mathrm{POT} \times 14 \mathrm{kt}$.

\section{CONCLUSIONS}

The NOvA experiment has finished construction and commissioning of both the $14 \mathrm{kt}$ far detector and the smaller 300 ton near detector. The upgrades to the Fermilab accelerator are complete and the beam power being delivered to the NOvA experiment is in the process of ramping up to full power. The NOvA detectors exhibit readout performance and noise rates have been demonstrated to meet or exceed their design specifications. This level of detector performance has allowed the experiment to identify a sample of neutrino candidate events in the early physics data. The sample demonstrates the capabilities of the NOvA detector and reconstruction algorithms to correctly detect neutrino interaction corresponding to all the topologies of interest for the oscillation analyses. Cosmic ray data from the first twelve months of detector commissioning and operations has been used to extensively validate the NOvA simulations and has been used for detector calibration. Data driven techniques based on the cosmic ray data have been used to obtain background estimates for the oscillation analyses. These studies have demonstrated a cosmic background rejection rate of 40M:1 for the $v_{e}$ analysis and 20M:1 for the $v_{\mu}$. Analysis tuning is now looking to perform similar data driven studies to tune the near detector rates and flavor decomposition for inclusion in the first oscillation analyses.

\section{ACKNOWLEDGMENTS}

The author acknowledges support for this research was carried out by the Fermilab scientific and technical staff. Fermilab is Operated by Fermi Research Alliance, LLC under Contract No. De-AC02-07CH11359 with the United States Department of Energy 